\title{
Posterior Spinal Surgery for Extradural Spinal Metastasis of Round Cell Tumor in the Lower Thoracal Region
}

\author{
Marleen' \\ Tjokorda GB Mahadewa' \\ I Wayan Juli Sumadi ${ }^{2}$ \\ 'Department of Neurosurgery, \\ Faculty of Medicine Udayana University, \\ Sanglah General Hospital \\ Bali, Indonesia \\ ${ }^{2}$ Department of Anatomical Pathology, \\ Faculty of Medicine Udayana University, \\ Sanglah General Hospital \\ Bali, Indonesia
}

\begin{abstract}
Spinal cord dysfunction caused by a spinal tumor is not always easy to treat since the tumor itself possesses variably etiology and low ability of nerve impairment to recover. The most common extradural tumor is derived from metastases, followed by other pathology such as plasmacytoma, lymphoma, etc. Since histopathologically they may present as round cell tumors, pre-operative data and intraoperative findings may help the clinician to establish appropriate diagnosis. This case report aims to describe the role of posterior spinal surgery and the reasoning of the selected approach in the treatment of spinal metastasis mimicking plasmacytoma in the lower thoracal region. We report 56 years old male who has myelopathy symptoms caused by lower thoracal extradural mass lesion, which histopathological study shows round cell tumor findings. Laminectomy and posterior stabilization were undertaken, and additional rotational flap was made to close sacral decubitus ulcer defect. Axial pain was improved and the patient discharged from the hospital with improved quality of life.
\end{abstract}

Keywords: Extradural tumor, metastasis, plasmacytoma, separate surgery, epidural spinal cord compression

\section{Introduction}

Extradural Spinal tumors can present as primary tumors or metastatic tumors. The spinal column involvement is common in spinal metastasis. Neurological deficits will profound when the tumor remarkably compresses the spinal cord. Despite surgery might not completely reverse the neurological deficits, the procedure routinely performed to alleviate pain, improve spinal instability, and for diagnostic purposes. ${ }^{1}$ Comprehensive management of tumor origin is mandatory, and it is not uncommon that spinal metastatic lesions are treated by chemotherapy and/ or radiation therapy. Benign or malignant are the type of primary tumors that can occur, and it is relatively rare. It can present as intradural or extradural neoplasms. Several pathology that categorized as primary malignant tumor are solitary plasmacytoma, osteosarcoma, Ewing's sarcoma, chondrosarcoma, chordoma, lymphoma, multiple myeloma. ${ }^{2}$ 
A group characteristic of male gender, sixth decade of age, extradural type of lesion and no evidence of other multiple bony involvement, give rise the possibility of metastatic lesion mimicking plasmacytoma. ${ }^{3,4}$ The primary malignant extradural spine lesion is not always easily differentiate with metastatic tumors. Collaboration among radiologic findings, intraoperative findings, and histopathological features will help clinicians to get an appropriate diagnosis.

Various surgical approach had been distinctly studied with numerous advantage and disadvantage. The surgical approach are divided into anterior and posterior approach in general. Posterior spinal surgery consist of posterior laminectomy with and without instrumentation, transpedicular corpectomy, and costotransversectomy. ${ }^{5}$ Surgical strategy must be tailored and individualized depend on clinical situations and surgeon familiarity. In this series, we will address laminectomy with instrumentation for managing patient who suffer from lower thoracal spinal metastasis.

\section{Case report}

Male 56 years old with myelopathy and thoracal pain. Symptoms occurred gradually within 6-months. There was no history of primary tumor or malignancies, trauma, and infections. Physical findings reveal axial thoracal pain and paraparesis (MRC Grade 1 on both lower extremity), paresthesia below $12^{\text {th }}$ thoracal level, urinary incontinence, and decubitus ulcer. Thoracolumbar MRI showed extradural mass involving left side vertebral body, pedicle, lamina, and facet joints, which compressing the exiting nerve root and spinal cord. The patient underwent decompressive laminectomy with posterior stabilization from Th12 until L2 level and gross total removal of the tumor. An additional rotational flap was applied to close the decubitus ulcer. Axial pain was improved postoperatively with persistent myelopathy (MRC Grade 1 on both lower extremity). The histopathological result showed features of round cell tumor with the possibility of metastatic small cell carcinoma.

\section{Discussion}

In the case development of primary and metastatic tumors, the spine is the most common site. Metastatic spinal tumors are the commonest among all spinal tumors. Spinal metastasis develops in 20 to $40 \%$ of all cancer patients. ${ }^{6}$ Thirty-one percent of autopsies of patients who died because malignant neoplasm harbor sites of spinal metastasis. ${ }^{7}$ Patients which develop symptomatic spinal metastasis are

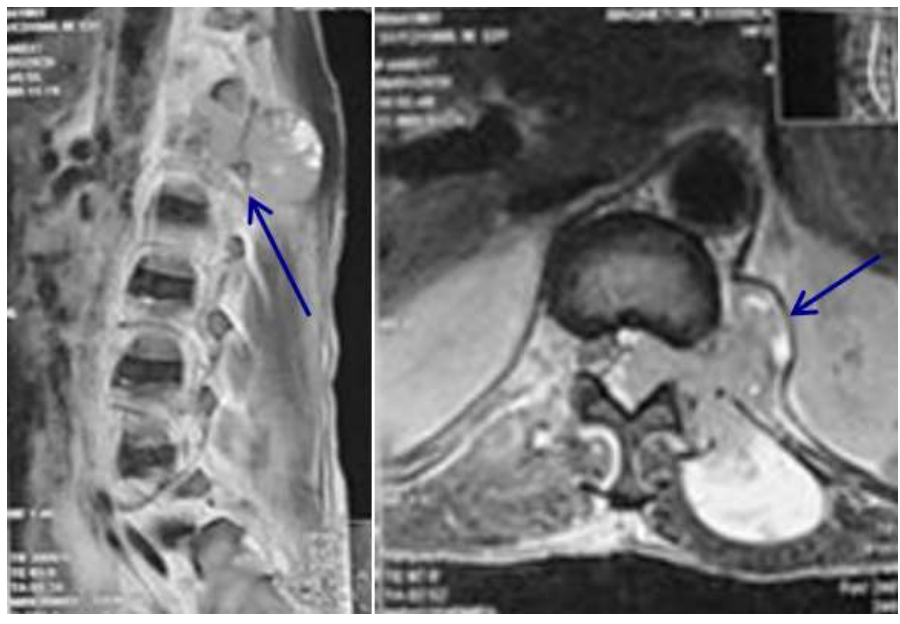

Figure 1. (Left, arrow) Sagital T1 contrast weighted image MRI shows heterogeneous enhanced mass lesion involving the posterior column of Th12 level. (Right, arrow) Axial T2 weighted image MRI shows dumbell shaped mass lesion involving the left posterior column of Th12 vertebrae

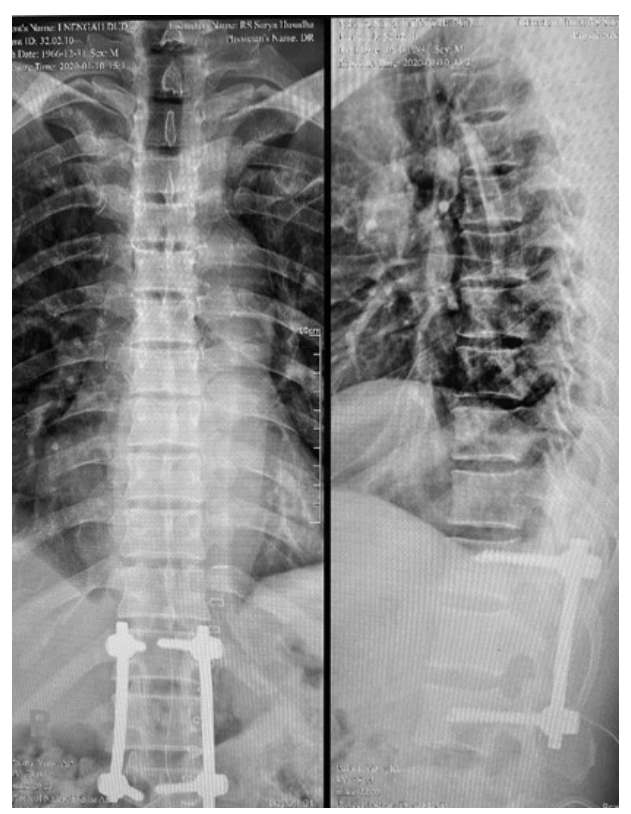

Figure 2. AP and lateral postoperative view of the thoracolumbar spine shows instrumentation was applied on Th12 to L2 vertebrae posteriorly. This procedure was performed to established stable thoracolumbar spine measure after posterior decompressive laminectomy was made

about $10 \%$ of all cancer patients, and $10 \%$ of them undergoing some form of surgical treatment. ${ }^{8-12}$ Furthermore, $75 \%$ of spinal metastases typically results from 1 of 5 malignancies: breast, lung, prostate, renal, or thyroid carcinoma. ${ }^{13}$

Since majority of primary spinal tumor are young age of onset (angiolipoma, tuberculoma, neurofibroma, aneurysmal bone cyst, ewing sarcoma, and neuroblastoma), and have specific site of occurrence (sacral origin for chordomas, skeletal muscle of rhabdomyosarcoma), we have to rule out another two possible 
diagnosis depend of patient preoperative findings, plasmacytoma and spinal metastasis. Plasmacytoma are B-cell lymphocyte neoplasms, very rare and affect either vertebral body or posterior element. ${ }^{14}$

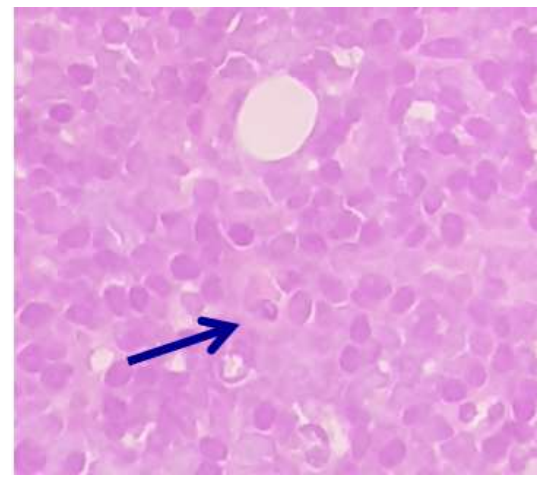

Figure 3. The proliferation of small round cells which diffusely arranged infiltrative among supporting and fatty tissue. The cells have uniformly small nuclei, inconspicuous nucleoli, and scant cytoplasm

Several algorithms were proposed by the previous study to determine which patient group will receive benefit from surgery or ideally treated conservatively. ${ }^{1}$ When surgery is indicated, the next challenging step is to select which approaches will give the most benefit for survival and patient clinical improvement. Among various posterior approaches available for the treatment of spinal metastasis, a study stressed that stand-alone laminectomy should be carefully considered since mechanical stabilization would be compromised in this group of patient. $^{15}$

Clinical assessment of extradural spinal tumors includes evaluation of myelopathy and epidural spinal cord compression (ESCC). ESCC score could be used in order to determine the need for surgery in terms of following radiation therapy. There are 6-points of ESCC grading scale; Grade 0: bone-only disease, Grade 1a: epidural impingement, without deformation of the thecal sac, Grade 1b: deformation of the thecal sac, without spinal cord impingement, Grade 1c: deformation of the thecal sac, with spinal cord abutment, without cord compression, Grade 2: spinal cord compression, with cerebral spinal fluid (CSF) visible around the cord, Grade 3: Spinal cord compression, no CSF visible around the cord. ${ }^{16}$

Surgery in spinal metastasis plays a crucial role since it provides diagnostic importance where the following treatment can be tailored based on it. Additional spinal cord decompression and control of spinal instability could be achieved by laminectomy and posterior instrumentation. Stand-alone laminectomy often argued that insufficiently treat spinal instability after decompression. The selected surgical approach must be tailored based on tumor extension in the spinal column. The anterior approach is preferred when the lesion exclusively involved the vertebral body, whereas the posterior or posterolateral approach is preferred when the lesion develops in the posterior spinal column. When radiosurgery becomes popularized recently, surgery also provides space between the tumor and spinal cord. This role of surgery is called as separation surgery, which was first time intoduced in 2000 as single-stage posterolateral trans-pedicle approach for spondylectomy, epidural decompression, and circumferential fusion for treatment of spinal metastasis. ${ }^{5}$

Posterior approach (posterior instrumentation followed by decompressive laminectomy) was selected in our case because the extradural lesion, with ESCC grade 3, incorporated a left-sided posterior column, which consists of facet joints, pedicles, and lamina. After meticulous removal of tumor mass by bilateral laminectomy and partial facetectomy, we apply a pedicle screw to stabilize the corresponding level of the spine $\left(12^{\text {th }}\right.$ Thoracal and $2^{\text {nd }}$ Lumbar Region).

\section{Conclusion}

Posterior instrumentation with laminectomy offers safe and mechanical stabilization advantage in metastatic spinal disease in lower thoracal region.

\section{Acknowledgment}

No acknowledgment

\section{References}

1. Sciubba DM, Gokaslan ZL. Diagnosis and management of metastatic spine disease. Surg Oncol. 2006;15:141-5. DOI: 10.1016/j.suronc.2006.11.002

2. Ciftdemir M, Kaya M, Selcuk E, et al. Tumors of the spine. World J Orthop. 2016;7(2):109-116. DOI: 10.5312/wjo.v7.i2.109

3. Mirzashahi B, Mazoochy H, Jamnani RK, et al. Contribution of surgery in solitary plasmacytoma of spine; a case report. Arch Bone Jt Surg. 2014;2(2):121-125

4. Rose P, Buchowski JM. Metastatic Disease in the Thoracic and Lumbar Spine: Evaluation and Management. J Am Acad Orthop Surg. 2011;19:37-48. DOI: 10.5435/00124635-201101000-00005

5. Molina C, Goodwin R, Abu-Bonsrah N, et al. Posterior approaches for symptomatic metastatic spinal cord compression. Neurosurg Focus. 2016;41(2):E11. DOI:10.3171/2016.5.FOCUS16129

6. Cobb CA 3rd, Leavens ME, Eckles N. Indications for nonoperative 
treatment of spinal cord compression due to breast cancer. $J$ Neurosurg. 1977;47(5):653-8. DOI: 10.3171/jns.1977.47.5.0653

7. Ortiz Gomez JA. The incidence of vertebral body metastases. Int Orthop. 1995; 19:309-11. DOI: 10.1007/bf00181116

8. Bell GR. Surgical treatment of spinal tumors. Clin Orthop Relat Res. 1997;335:54-63.

9. Bilsky MH, Lis E, Raizer J, et al. The diagnosis and treatment of metastatic spinal tumor. Oncologist. 1999;4:459-69. DOI:10.1634/THEONCOLOGIST.4-6-459

10. Sundaresan N, Digiacinto GV, Hughes JE, et al. Treatment of neoplastic spinal cord compression: Results of a prospective study. Neurosurgery. 1991;29:645-50. DOI: 10.1097/00006123199111000-00001

11. Walsh GL, Gokaslan ZL, McCutcheon IE, et al. Anterior approaches to the thoracic spine in patients with cancer: Indications and results. Ann Thorac Surg. 1997;64:1611-18. DOI: 10.3171/jns.1998.89.4.0599
12. York JE, Walsh GL, Lang FF, et al. Combined chest wall resection with vertebrectomy and spinal reconstruction for the treatment of Pancoast tumors. $J$ Neurosurg. 1999;91(1Suppl):74-80. DOI: 10.3171/spi.1999.91.1.0074

13. Harrington $\mathrm{KD}$. The use of methylmethacrylate for vertebral-body replacement and anterior stabilization of pathological fracturedislocations of the spine due to metastatic malignant disease. $J$ Bone Joint Surg Am. 1981;63:36-46. DOI:10.2106/00004623-19816301000005

14. Sharma, Gopal \& Khadka, Nilam \& Jha, Rajiv \& Adhikari, Dhurba \& Bista, Prakash. (2016). Extradural Spinal Tumors: Report of 36 Cases and Review of Literature. Nepal Journal of Neuroscience. 2016:12(1):3-10. DOI:10.3126/njn.v13i1.15905

15. Bilsky MH, Laufer I, Fourney DR, et al. Reliability analysis of the epidural spinal cord compression scale. J Neurosurg Spine. 2010;13(3):324-8. DOI: 10.3171/2010.3.spine09459

16. Bilsky M, Smith M. Surgical approach to epidural spinal cord compression. Hematol Oncol Clin North Am. 2006;20(6):1307-17. DOI: 10.1016/j.hoc.2006.09.009 